\title{
Clinical Study of Cardiac Diseases during Pregnancy in a Tertiary Care Center, Kakinada
}

\author{
Indira Surya Kumari Kothapalli ${ }^{1}$, Akkamamba Basa² ${ }^{2}$ Neela Aparna Medapati ${ }^{3}$, Soumini Gangaraju ${ }^{4}$ \\ ${ }^{1}$ Assistant Professor, Department of Obstetrics and Gynaecology, Rangaraya Medical College, \\ Kakinada, Andhra Pradesh, India. \\ ${ }^{2}$ Associate Professor, Department of Obstetrics and Gynaecology, Rangaraya Medical College, \\ Kakinada, Andhra Pradesh, India. \\ ${ }^{3} 2^{\text {nd }}$ Year Postgraduate Student, Department of Obstetrics and Gynaecology, Rangaraya Medical College, \\ Kakinada, Andhra Pradesh, India. \\ ${ }^{4}$ Professor, Department of Obstetrics and Gynaecology, Rangaraya Medical College, \\ Kakinada, Andhra Pradesh, India.
}

\section{ABSTRACT}

\section{BACKGROUND}

Cardiovascular abnormalities are considered to be the most important non obstetric cause of morbidity. The primary objective of the present study is to assess the effect of heart diseases in pregnancy and its outcome. If diagnosed early and managed properly with multidisciplinary team approach, collaboration of trained obstetrician, cardiologist, anaesthetist, paediatrician and nurse, it results in a successful outcome for mother and child in majority of cases. The primary objective of the present study is to assess the effect of heart diseases on pregnancy and its outcome

\section{METHODS}

The study was carried out in a tertiary care centre, GGH KKD over the period January 2018-January 2019. All antenatal patients having cardiac diseases either previously diagnosed or diagnosed during antenatal visits, early postnatal patients with booked antenatal visits, referrals with cardiac diseases, emergency admissions, patients who developed cardiac complications during peripartum period were included in the study. Data was statistically analysed SPSS 16.

\section{RESULTS}

Out of a total of 10936 admissions in our hospital during the study period, there were 135 admissions with heart disease complicating pregnancy (1.23\%). 100 of them were included in the study. The RHD (40\%) was the principal cause of heart diseases among all the pregnancies. Second common cause is congenital heart diseases. Of these, $26 \%$ underwent cardiac surgery. Peripartum cardiomyopathy was found in $6 \%$ of patients.

\section{CONCLUSIONS}

This study concluded that pre pregnancy diagnosis, counselling appropriate referral, routine antenatal supervision, and delivery at an equipped centre improve the foetomaternal outcome in pregnancy with heart disease.

\section{KEY WORDS}

Rheumatic Heart Diseases, Congenital Heart Diseases, Caesarean Section
Corresponding Author: Dr. Akkamamba Basa,

Manasa Chola Apartments FFF2, Near Subbiah Hotel, Ramaraopeta, Kakinada, Andhra Pradesh, India. E-mail: akkamamba001@gmail.com

DOI: 10.14260/jemds/2019/569

Financial or Other Competing Interests: None.

How to Cite This Article: Kothapalli ISK, Basa A, Medapati NA, et al. Clinical study of cardiac diseases during pregnancy in a tertiary care center, Kakinada. J. Evolution Med. Dent. Sci. 2019;8(33):2612-2616, $10.14260 /$ jemds/2019/569

Submission 31-05-2019, Peer Review 01-08-2019, Acceptance 07-08-2019, Published 19-08-2019. 


\section{BACKGROUND}

Cardiac diseases complicate about $1 \%{ }^{1}$ of pregnancies in women. Cardiac diseases in pregnancy is broadly divided into congenital $\{\mathrm{CHD}\}$, and acquired. The proportion of women of child-bearing age with CHD, surgically treated has increased substantially in recent decades due to improved surgical and cardiac care. In developing countries, RHD is the commonest type. Among all presentations of Rheumatic heart disease (RHD), Mitral Stenosis (MS) is the predominant lesion accounting for nearly 3 quarters of all cases.

"Pregnancy is a natural stress test," as opined by James martin** M.D., chair of the Pregnancy and Heart Disease Task Force. "The cardiovascular system must undergo major changes to its structure to sustain tremendous increases in blood volume. So it is critical to identify the risk factors beforehand, so that a woman's care can be properly managed throughout the pregnancy and a detailed delivery plan can be developed through shared decision making between the patient and provider. Moreover, we must think of heart disease as a possibility in every pregnant or postpartum patient we see to detect and treat at-risk mothers." The presence of maternal heart diseases effects on the foetus in a many number of ways. The risk of spontaneous miscarriages and therapeutic abortions increases in women with heart diseases $^{2}$. Women with cardiovascular disease should be advised that pregnancy can contribute to a decline in cardiac status, risk of maternal mortality or morbidity, and foetal risks such as preterm birth. Caesarean delivery is usually limited to obstetrical indications, and considerations are given for the specific cardiac lesions like Aortic dissection, Marfan's syndrome with dilated aortic root, overall maternal condition, and availability of experienced anaesthesia personnel and hospital capabilities. Forceps or vacuum extraction to cut short the second stage of labour to shorten and ease delivery, patients at intermediate or high risk may require monitoring for at least 72 hours postpartum. Cardiovascular abnormalities are considered most important non obstetric cause of morbidity and are a prominent reason for obstetrical intensive care unit admissions ${ }^{3}$. The circulatory changes of pregnancy in the presence of maternal heart diseases may result in adverse consequences even death of mother or foetus. Pregnancy is a challenge to women with heart diseases because of $50 \%$ increase in plasma volume and 6 -fold increase in risk of thrombosis 4 . Importantly, cardiac output increases approximately 40 percent during pregnancy. Almost half of this total takes place by 8 weeks' gestation and is maximal by midpregnancy. ${ }^{5}$ According to the National Centre for Health Statistics, almost half of adults aged 20 and older have at least one risk factor for cardiovascular disease. The rising prevalence of cardiovascular diseases complicating pregnancy is likely multifactorial and includes the higher rates of obesity, hypertension, and diabetes. ${ }^{6}$ Problems should be identified early and treated aggressively, especially pregnancy induced hypertension, hyperthyroidism, infection, and anaemia. In developing countries, a large number of women become pregnant prior of seeking therapeutic intervention for cardiac lesions and many of them are only diagnosed with heart diseases during pregnancy. ${ }^{7}$ Detailed assessment of patient throughout pregnancy may lead to initial discovery of heart diseases. The primary objective of the present study is to assess the effect of heart diseases in pregnancy and its outcome. A pregnancy heart team should conduct ongoing evaluation of all pregnant and postpartum women with known or suspected cardiovascular disease." The pregnancy heart team should have a comprehensive plan established for the pregnancy, delivery, and postpartum period. The plan should include the review of cardiac medication safety for the mother and the foetus, and the risk to the foetus from congenital and genetic conditions."

The first 10 ACOG recommendations drawn from consensus and expert opinion feature advisories for maternal health as well as foetal and neonate care-

1.Knowledge: Clinicians should be familiar with signs and symptoms of cardiovascular disease.

2.Assessment: Ideally, a cardiologist should evaluate women with cardiovascular disease before pregnancy or as early as possible during the pregnancy for diagnosis, assessment of the effect pregnancy will have on cardiovascular conditions, risks to the woman and foetus, and treatment of underlying cardiac conditions.

3.Patient Management: Women with cardiovascular disease risk should be managed through pregnancy and the postpartum period by a pregnancy heart team.

4.Patient Engagement: Women with cardiovascular disease should be advised that pregnancy can contribute to a decline in cardiac status, risk of maternal mortality or morbidity, and foetal risks such as preterm birth.

5.Individualized Care: To support the mother's decision making, the care team should take a personalized approach that accounts for maternal and foetal hazards linked to specific cardiac disorders and the patient's pregnancy plans.

6.Assessment Tool: The California Improving Health Care Response to Cardiovascular Disease in Pregnancy and Postpartum toolkit should be used to assess all pregnant women for cardiovascular disease.

7.0ngoing Evaluation: A pregnancy heart team should conduct ongoing evaluation of all pregnant and postpartum women with known or suspected cardiovascular disease.

8.Testing Protocols: For women with cardiovascular disease and symptoms such as shortness of breath, chest pain, or palpitations, testing of cardiac status during pregnancy and the postpartum period is warranted.

9.ECG Testing: Pregnant and postpartum women with known or suspected congenital heart disease, valvular and aortic disease, cardiomyopathies, and a history of cardiotoxic chemotherapy should have echocardiogram examination.

10. Foetal Testing: For women with congenital heart disease, there should be foetal echocardiography. Conversely, when congenital heart disease is found in a foetus or neonate, screening for parental congenital heart disease could be warranted.

Successful outcome for mother and child can occur in majority of cases if diagnosed early and managed properly with multidisciplinary team approach, collaboration of trained obstetrician, cardiologist, anaesthetist and paediatrician and nurse. $^{8}$

\section{METHODS}

The study was an observational study carried out in a tertiary care centre, GGH KKD over a period of January 2018 to January 2019. All antenatal patients having cardiac diseases diagnosed 
either previously or diagnosed at booked antenatal visits, referrals with cardiac diseases, early postnatal patients, emergency admissions patients who developed cardiac complications during peripartum period were included in the study. Informed consent is taken from the patients. There was an incidence of cardiac diseases was $1.7 \%$ in our hospital. Most of the women belong to low socioeconomic class. Detailed history of cardiovascular examination was performed to determine the type of lesion, patient suspected of cardiac lesions were evaluated by cardiologist to diagnose specific cardiac lesions. Risk assessment done with functional status (NYHA class III or IV) or cyanosis, Left ventricular systolic dysfunction (Ejection fraction $<0.40$ ), Left heart obstruction (Mitral valve area $<2.0 \mathrm{~cm}^{2}$, aortic valve area $<1.5 \mathrm{~cm}^{2}$, or peak left ventricular outflow tract gradient $>30 \mathrm{~mm} \mathrm{Hg}$ ) A cardiac event (Arrhythmia, stroke, transient ischemic attack, or pulmonary oedema).

\begin{tabular}{|} 
RESULT S \\
\begin{tabular}{|c|c|}
\hline \multicolumn{1}{|c|}{} \\
\hline Primi & 39 \\
\hline G2 & 41 \\
\hline G3 & 16 \\
\hline$>3$ & 3 \\
\hline \multicolumn{2}{|c|}{ Parity of the Mother } \\
\hline
\end{tabular}
\end{tabular}

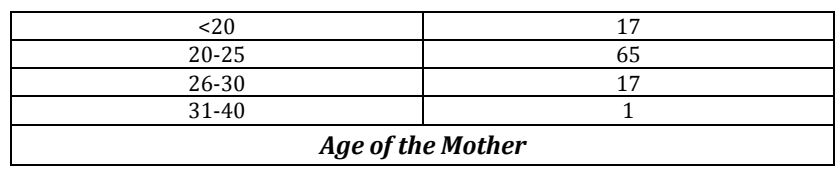

\begin{tabular}{|c|c|c|}
\hline & No. & Percentage \\
\hline Valvular heart disease & 50 & 50 \\
\hline Congenital heart disease with cardiac surgery & 17 & 17 \\
\hline CHD without prior cardiac surgery & 13 & 13 \\
\hline Peripartum cardiomyopathy & 6 & 6 \\
\hline Miscellaneous & 14 & 14 \\
\hline \multicolumn{2}{|c|}{ Types of Cardiac Lesions } \\
\hline
\end{tabular}

\begin{tabular}{|c|c|}
\hline Mode of Delivery & No. \\
\hline Vaginal & 56 \\
\hline Instrumental & 11 \\
\hline LSCS & 29 \\
\hline Abortion & 4 \\
\hline Mode of Delivery \\
\hline
\end{tabular}

\begin{tabular}{|c|c|}
\hline Small for Gestational Age & $\mathbf{3 5}$ \\
\hline IUFD & - \\
\hline Neonatal Deaths & 3 \\
\hline Term Babies & 58 \\
\hline & No. \\
\hline Birth Weight & - \\
\hline$<1.5$ Kgs. & 34 \\
\hline $1.5-2.5$ Kgs. & 62 \\
\hline$>2.5$ Kgs. \\
\hline \multicolumn{2}{|c|}{ Details of the Babies } \\
\hline
\end{tabular}

\begin{tabular}{|c|c|}
\hline & No. \\
\hline None & 47 \\
\hline Digoxin & 4 \\
\hline Diuretic & 10 \\
\hline Anti-arrhythmic & 3 \\
\hline Anti-coagulant & 5 \\
\hline Prior heart failure & 5 \\
\hline Prior cardiac surgery & 26 \\
\hline Receiving Cardiac Medication \\
\hline
\end{tabular}

Out of total 10936 admissions in our hospital in year, 135 of women had pregnancy complicated by diagnosed heart diseases (1.7\%). 100 patients were included in the study. Majority age of patients ranged in the age group of 20-25 years. In this study most of the patients were second gravida. Out of 100 cases valvular heart diseases accounts for $50 \%$ and among them the RHD heart diseases are more prevalent. The RHD (40\%) was the principal cause of heart diseases among all the pregnancies. Among the RHD Mitral stenosis is the most common. The next most common heart disease was congenital heart diseases (30\%).Of all the congenital heart diseases atrial septal defect (ASD) is the most common and majority of them were surgically corrected. Of these $26 \%$ underwent prior cardiac surgery. Peripartum cardiomyopathy was found in $6 \%$ of patients. The aetiology of peripartum cardiomyopathy remains unknown, and proposed causes include viral myocarditis, abnormal immune response to pregnancy, aberrant response to the greater hemodynamic burden of pregnancy, hormonal interactions, malnutrition, inflammation, and apoptosis.

Valvular Lesions-

- $\quad$ Trivial MR- 10.

- $\quad$ MS- 30

- $\quad$ TR- 9

- $\quad$ Mitral valve prolapse- 1

Prior Cardiac and Congenital Lesions-

- $\quad$ ASD- 23

- VSD- 4

- $\quad$ PDA- 1

- Tetralogy of Fallot- 2

Miscellaneous-

- Cardiomyopathy- 4

- Sinus arrhythmia- 9

- $\quad$ Left ventricular hypokinesia- 1

Among the cardiac surgeries ASD closure was done in 10 patients, VSD closure were done in 3 patients, Balloon valvuloplasty was done in 6 patients. Tetralogy of Fallot was corrected in 2 patients, Mitral valve replacement was done in 5 patients. The aetiology of cardiomyopathy is idiopathic in most of the cases. Pregnant and postpartum women with known or suspected congenital heart disease, valvular and aortic disease, cardiomyopathies, and a history of cardiotoxic chemotherapy had echocardiogram examination. Patients included in the study group were categorised based on their symptoms into NYHA classification:

NYHA Class I: 54 patients. NYHA Class II: 39 patients. NYHA Class III: 5 patients. NYHA Class IV: 2 patients.

Mode of delivery was vaginal in majority of cases 56\%. Epidural analgesia was given to $30 \%$ of those delivered vaginally, $29 \%$ delivered by LSCS, $11 \%$ by outlet forceps delivery. Out of 100 deliveries 8 were preterm deliveries. Medical termination of pregnancy was done in 4 patients. 65 babies weighed $>2.5 \mathrm{kgs}$., and 35 babies were between 1.5 and 2.5 kgs. Among the babies there were 18 NICU admissions 3 neonatal deaths. Among all the babies born alive none had congenital heart diseases. 35 Babies were small for gestational 
age. $5 \%$ of the patients admitted has prior cardiac failure 22 patients were on cardiac medication. Among them 4 were on Digoxin, 10 were on diuretic, 5 were on Warfarin and 3 on anti arrhythmics. The morbidity with which the patients were admitted in ICU was acute onset of breathlessness.

\section{DISCUSSION}

Reducing maternal mortality is a World Health Organization (WHO) global health goal. Although maternal deaths due to haemorrhage and infection are declining, those related to heart disease are increasing and are now the most important. This study was conducted at department of obstetrics and gynaecology, government general hospital Kakinada in a total of 100 women a tertiary centre with rural referrals. This study aimed at assessment of maternal and neonatal complications associated with cardiac diseases in pregnancy. In the present study, the prevalence of $1.7 \%$, comparable to $1 \%$ in Bhatla $\mathrm{N}$, et $\mathrm{al}^{1}$ differs from the $4.5 \%$ in a study conducted by Puri et al ${ }^{9}$. In the current study majority of the patients were in the age group of 20-30 years (65\%) and most of them are second gravidae. In Vidyadhare ${ }^{10}$ study $70 \%$ were either primigravida or primipara. In an analysis of maternal mortality in the United States between 2011 and 2013, the causes previously responsible for most maternal deaths- haemorrhage, hypertensive disorders, and Embolism continued to show declining rates. In contrast, deaths attributable to cardiovascular diseases were responsible for approximately 26 percent of all pregnancy-related deaths. Using the Registry of Pregnancy and Cardiac Disease, the maternal Mortality rate was 1.4 percent in women with a mechanical heart valve and 1. 5 percent in women with a tissue heart valve. In the current study RHD (40\%) was the principal cardiac lesion and mitral stenosis was the most common cardiac lesion. Rheumatic fever is uncommon in the United States because of less crowded living conditions, penicillin availability, and evolution of non rheumatogenic streptococcal strains. Still, it remains the chief cause of serious mitral valvular disease in women of childbearing age in the non-industrialized world Thus the current study indirectly indicates inadequate treatment of streptococcal infections in childhood and adolescence. Echocardiography was helpful for early and accurate evaluation of cardiac lesions. In general, vaginal delivery is preferred, and labour induction is usually safe. ${ }^{11}$ Mortality in pregnant females with cardiac diseases is mainly due to cardiac failure and pulmonary oedema. Cardiovascular decompensation during labour may manifest as pulmonary oedema with hypoxia or as hypotension, or both. Fluid mobilized into the intravascular compartment and reduced peripheral vascular resistance place higher demands on myocardial performance. Therefore, meticulous care is continued into the puerperium. Postpartum haemorrhage, anaemia, infection, and thromboembolism are much more serious complications with heart disease, sepsis and severe preeclampsia cause or worsen pulmonary oedema because of endothelial activation and capillary-alveolar leakage. Pregnant women with Eisenmenger syndrome tolerate hypotension poorly, and death usually is caused by right ventricular failure with cardiogenic shock. Eisenmenger syndrome is considered to be an absolute contraindication to pregnancy.

"The rise we're seeing in maternal deaths is largely due to acquired cardiac disease in pregnancy," said Hollier. "Most of these deaths are preventable, but we are missing opportunities to identify risk factors prior to pregnancy and there are often delays in recognizing symptoms during pregnancy and postpartum, particularly for black women. The new guidance clearly delineates between common signs and symptoms of normal pregnancy versus those that are abnormal and indicative of underlying cardiovascular disease. As clinicians, we need to be adept at distinguishing between the two if we're going to improve maternal outcomes." In developed countries infective Rheumatic lesions are decreased but still common in India where they can be worked out for pregnancy. But congenital, atherosclerotic, peripartum cardiomyopathies are on rise with improved diagnostic techniques. So pre pregnancy screening definitely improves foetomaternal outcome screening. Mortality in pregnant females with cardiac diseases is mainly due to cardiac failure and pulmonary oedema. In our study out of 100 patients 2 were died due to cardiac failure and sepsis which was comparable to the study done by Saima Salam ${ }^{12}$ and Kamat. ${ }^{13}$ Both were of term gestation and one was delivered vaginally developed peripartum cardiomyopathy and the other a known case of congenital heart disease delivered through caesarean section.

\section{CONCLUSIONS}

Reducing maternal mortality is a World Health Organization (WHO) global health goal. Although maternal deaths due to haemorrhage and infection are declining, those related to heart disease are increasing and are now the most important cause. The aim is to define contemporary diagnosis-specific outcomes in pregnant women with heart disease. Early diagnosis of heart disease, regular antenatal checkup, institutional delivery, limiting family size can reduce the maternal and perinatal mortality and morbidity associated with heart disease. Counselling is an important aspect of management of the cardiac patient planning a pregnancy. Ideally, a team approach with obstetrician and cardiologist working together should help the patient to make an informed decision for a good maternal and foetal outcome.

\section{REFERENCES}

[1] Bhatla N, Lal S, Behera G, et al. Cardiac disease in pregnancy. Int J Gynecol Obstet 2003;82(2):153-9.

[2] Siu SC, Colman JM, Sorensen S, et al. Adverse neonatal and cardiac outcomes are more common in pregnant women with cardiac disease. Circulation 2002;105(18):2179-84.

[3] Small MJ, James AH, Kershaw T, et al. Near-miss maternal mortality: cardiac dysfunction as the principal cause of obstetric intensive care unit admissions. Obstet Gynecol 2012;119(2 Pt 1):250-5.

[4] Steer P. Heart disease in pregnancy. Women's health medicine. Med Prob Pregnancy 2005;2(2):18-21. 
[5] Capeless EL, Clapp JF. Cardiovascular changes in early phase of pregnancy. Am J Obstet GynecoI 1989;161(6 Pt 1):1449-53.

[6] Klingberg, Brekke HK, Winkvist A, et al. Parity, weight change and maternal risk of cardiovascular events. Am J Obstet Gynecol 2017;216(2):172.e1-e15.

[7] Sawhney H, Aggarwal N, Suri V, et al. Maternal and perinatal outcome in rheumatic heart disease. Int $\mathrm{J}$ Gynaecol Obstet 2003;80(1):9-14.

[8] Trinidad D, Cox RA. Heart diseases during pregnancy. PR Health Sci J 2006;25:259-65.

[9] Puri S, Bharti A, Puri S, et al. Maternal heart disease and pregnancy outcomes. JK Sci 2013;15(1):7-10.
[10] Bangal VB, Singh RK, Shinde KK. Clinical study of heart diseases complicating pregnancy. IOSR 2012;2(4):25-8.

[11] Thurman R, Zafar N, Sayyar P, et al. Labour profile and outcomes in pregnant women with heart disease. Abstract No. 799. Am J Obstet Gynecol 2017;216(1):S459S60.

[12] Salam S, Mushtaq S, Mohi-ud-Din K, et al. Maternal and foetal outcome in pregnancy with heart disease in tertiary care hospital in India. Int J Reproad Contracept Obstet Gynecol 2017;6(9):3947-51.

[13] Kamat AV, Dama S. Clinical study of cardiac diseases during pregnancy. Int J Reproad Contracept Obstet Gynecol 2016;5(3):855-9. 